\title{
Permeation of Toluene into (Polypropylene/Acrylonitrile-co-butadiene Rubber) immiscible blend
}

\author{
Y. HAIRCH, A. EL AFIF \\ Physics Department, Chouaïb Doukkali University, El Jadida, Morocco
}

Corresponding author: alielafif2005@yahoo.fr

\begin{abstract}
This study examines the unsteady permeation process of a solvent through a mixture of two immiscible polymers with a complex dividing interface. The mathematical model consists of three nonlinear PDEs that incorporate the coupling arising between mass transport and the dynamic changes of the blend internal structure. In addition to the solvent mass fraction c, we choose, as additional state variables, the conformation tensor $\mathbf{m}$ to characterize the macromolecular chains while the embedded interface is described by a scalar $\mathbf{Q}$ and a traceless second-order tensor q respectively for the size and shape anisotropy of the area. We extend the Fick's first law by two new non-Fickian terms. Among the obtained results, we present the transient permeation mass flux profiles of toluene through PP/NBR blend system at different compositions and predict the corresponding time lag.
\end{abstract}

Keywords: Non-Fickian, permeation, Interface, conformation, time lag.

\section{Introduction}

Diffusion of small organic molecules into polymeric systems is of great importance in many industrial applications such as barrier, packaging and protective coatings. In this paper, the study focuses on the permeation process of a solvent through a blend composed of two immiscible polymers separated by a complex interface. Note that Fick's laws suffer from several shortcomings in particular when the system under consideration possesses a dynamically active internal structure. Thereby such changes have to be included into a comprehensive mathematical formulation of mass transport [1-2]. From the thermodynamic viewpoint, we require that the driving force for diffusion is the gradient of the chemical potential rather than that of the concentration as stipulated by Fick's law. Moreover, we use additional structural variables to track down the changes occurring in the macromolecular chains as well as at the embedded interface while undergoing diffusion. Here, we limit the study to the isothermal situation, under the mechanical equilibrium and in the absence of an applied flow. The model consists of a set of nonlinear partial differential equations whose dimensionless form includes two Deborah numbers and two coupling constants. We investigate in particular the permeation process of toluene into a PP/NBR immiscible blend at different compositions and calculate the time lag necessary to reach the steady state.

\section{Governing equations}

The set of the independent state variables used in this investigation, and defined earlier, is $(c, Q, q, m)$. In the absence of chemical reactions and flow, the solvent continuity equation can be written under the following form [1-2]:

$$
\frac{\partial c}{\partial t}=\frac{\partial}{\partial x}\left(D(c)\left(\frac{\partial c}{\partial x}+A \frac{\partial Q}{\partial x}+B \frac{\partial m}{\partial x}\right)\right)
$$

Where $\mathrm{t}$ is time and $x$ refers to the Eulerian direction of diffusion since mass transport into thin films is generally unidirectional. In Eq. (1), $D(c)=D_{0} \exp \left(k_{D} c\right)$ is the diffusivity coefficient and $k_{D}$ is a material plasticization parameter. Moreover, this equation involves two coupling functionals :

$$
\begin{aligned}
& A=(\partial \mu / \partial Q) /(\partial \mu / \partial c) \\
& B=(\partial \mu / \partial m) /(\partial \mu / \partial c)
\end{aligned}
$$

where $\mu$ is the solvent chemical potential. The governing equation for the size density of the interface area is:

$$
\begin{aligned}
& \frac{\partial Q}{\partial t}+\frac{1-c}{\tau_{Q q}}\left(Q-Q_{e q}\right)= \\
& -\frac{D(c)}{1-c} \frac{\partial}{\partial x}\left(\frac{\partial c}{\partial x}+A \frac{\partial Q}{\partial x}+B \frac{\partial m}{\partial x}\right) \frac{\partial Q}{\partial x} \\
& -\left(q+\frac{Q}{3}\right) \frac{\partial}{\partial x}\left(\frac{D(c)}{1-c}\left(\frac{\partial c}{\partial x}+A \frac{\partial Q}{\partial x}+B \frac{\partial m}{\partial x}\right)\right)
\end{aligned}
$$


where $\tau_{\mathrm{qQ}}$ is the interface relaxation time and $Q_{e q}$ is the final equilibrium size density of the interface. The governing equation for the anisotropy density of the shape of the interface is as:

$$
\begin{aligned}
& \frac{\partial q}{\partial t}+\frac{1-c}{\tau_{Q q}}\left(q-q_{e q}\right)= \\
& -\frac{D(c)}{1-c}\left(\frac{\partial c}{\partial x}+A \frac{\partial Q}{\partial x}+B \frac{\partial m}{\partial x}\right) \frac{\partial q}{\partial x} \\
& -\left(q-\frac{q^{2}}{Q}+\frac{4 Q}{9}\right) \frac{\partial}{\partial x}\left(\frac{D(c)}{1-c}\left(\frac{\partial c}{\partial x}+A \frac{\partial Q}{\partial x}+B \frac{\partial m}{\partial x}\right)\right)
\end{aligned}
$$

where $q_{e q}$ is the final equilibrium anisotropy density. Finally, the time evolution equation for the conformation of the macromolecular chains is:

$$
\begin{aligned}
& \frac{\partial m}{\partial t}+\frac{1-c}{\tau_{m}}\left(\left(1-c\left(2-c_{e q}\right)\right) \frac{m}{K_{B} T / k}-1\right)= \\
& -\frac{D(c)}{1-c}\left(\frac{\partial c}{\partial x}+A \frac{\partial Q}{\partial x}+B \frac{\partial m}{\partial x}\right) \frac{\partial m}{\partial x} \\
& +m \frac{\partial}{\partial x}\left(\frac{D(c)}{1-c}\left(\frac{\partial c}{\partial x}+A \frac{\partial Q}{\partial x}+B \frac{\partial m}{\partial x}\right)\right)
\end{aligned}
$$

where $\tau_{\mathrm{m}}$ is the conformation relaxation characteristic time, $\mathrm{k}_{\mathrm{B}}$ is the Boltzmann constant, $\mathrm{T}$ is the absolute constant temperature and $\mathrm{H}$ is a characteristic elastic constant. We Scale the model equations using the following transformations:

$$
\begin{aligned}
& X=x / L_{0} \quad \theta=\mathrm{t} / \tau_{d} \\
& \widehat{\mathrm{c}}=\mathrm{c} / \mathrm{c}_{\text {eq }}, \widehat{\mathrm{Q}}=\mathrm{Q} / \mathrm{Q}_{0}, \widehat{\mathrm{q}}=\mathrm{q} / \mathrm{Q}_{0}, \widehat{\mathrm{m}}=\mathrm{m} /\left(K_{B} T / k\right)
\end{aligned}
$$

where $L_{0}$ stands for the initial thickness of the blend and $\tau_{d}$ is the diffusion characteristic time scale. $Q_{0}$ is the amount of the size density at the dry state. Scaling leads to the emergence of two coupling constants:

$$
\begin{aligned}
& g_{A}=\frac{\Gamma_{0} Q_{0} \Omega_{s}}{R T} \\
& g_{B}=\frac{G_{0} \Omega_{s}}{2 R T}
\end{aligned}
$$

where $\mathrm{R}$ is the gas constant, $\Omega_{s}$ is the solvent molar volume, $\Gamma_{0}$ is the interfacial tension and $G_{0}$ is the blend elasticity modulus. Moreover, the normalized equations involve two diffusion-Deborah numbers:

$$
\begin{aligned}
& D e_{Q q}=\frac{\tau_{Q q}}{\tau_{d}} \\
& D e_{m}=\frac{\tau_{m}}{\tau_{d}}
\end{aligned}
$$

The former is for the interface and the latter is for the conformation. Recall that the Deborah number compares the importance of the viscoelastic effects of the polymeric blend with mixing. When De is comparable to the unit, diffusion deviates from the predictions of the parabolic Fickian theory and becomes non-Fickian or viscoelastic.

\section{Results and discussion}

We discretize and solve the normalized governing equations by using the finite difference method which allows us to obtain profiles of the mass fraction $\hat{c}$, the size density $\widehat{Q}$ and the anisotropy density $\widehat{q}$ of the interface as well as the conformation $\widehat{m}$. We are also capable of calculating the swelling of the blend and the distribution of the stresses created by the penetration of the solvent. The boundary conditions used for solving numerically the dimensionless governing equations are:

$$
\begin{array}{ll}
\widehat{\mathrm{c}}(\mathrm{X}=1, \theta)=0 & \widehat{\mathrm{Q}}(\mathrm{X}=1, \theta)=1 \\
\widehat{\mathrm{q}}(\mathrm{X}=1, \theta)=0 & \widehat{\mathrm{m}}(\mathrm{X}=1, \theta)=1
\end{array}
$$

The mass flux is calculated at the exit using the following non-Fickian extended expression:

$\tilde{J}=-\left.\widehat{D}(c)\left(\frac{\partial \widehat{c}}{\partial X}+g_{A} \widetilde{A} \frac{\partial \widehat{Q}}{\partial X}+g_{B} \widetilde{B} \frac{\partial \widehat{m}}{\partial X}\right)\right|_{X=1}$

which involves the normalized functionals: $\widehat{\mathrm{D}}(\mathrm{c})=$ $\exp \left(k_{D}(c-1)\right), \widetilde{\mathrm{A}}=A / g_{A}$ and $\widetilde{\mathrm{B}}=B / g_{B}$.

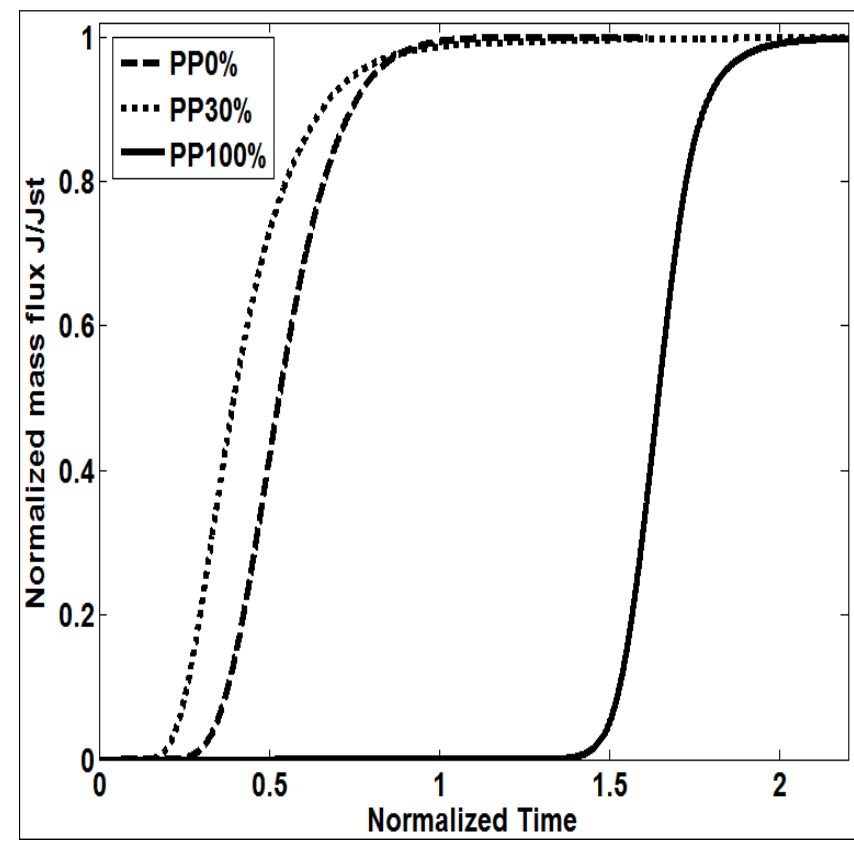

Figure 1: Unsteady normalized permeation mass flux of toluene into PP/NBR at different blend compositions $J / J_{s t} v s$ normalized time. 
Figure 1 shows the normalized solvent mass flux $\tilde{\mathrm{J}}=\left(\mathrm{J} / \mathrm{J}_{\mathrm{st}}\right)$ versus normalized time, where $\left(\mathrm{J}_{\mathrm{st}}\right)$ is the steady mass flux of toluene. We observe that the time necessary to reach the steady state is greater in the blend than in the pure NBR. That time becomes significant in the particular case of the pure PP. This is certainly due to its high degree of cristallinity as reported in Ref [3].

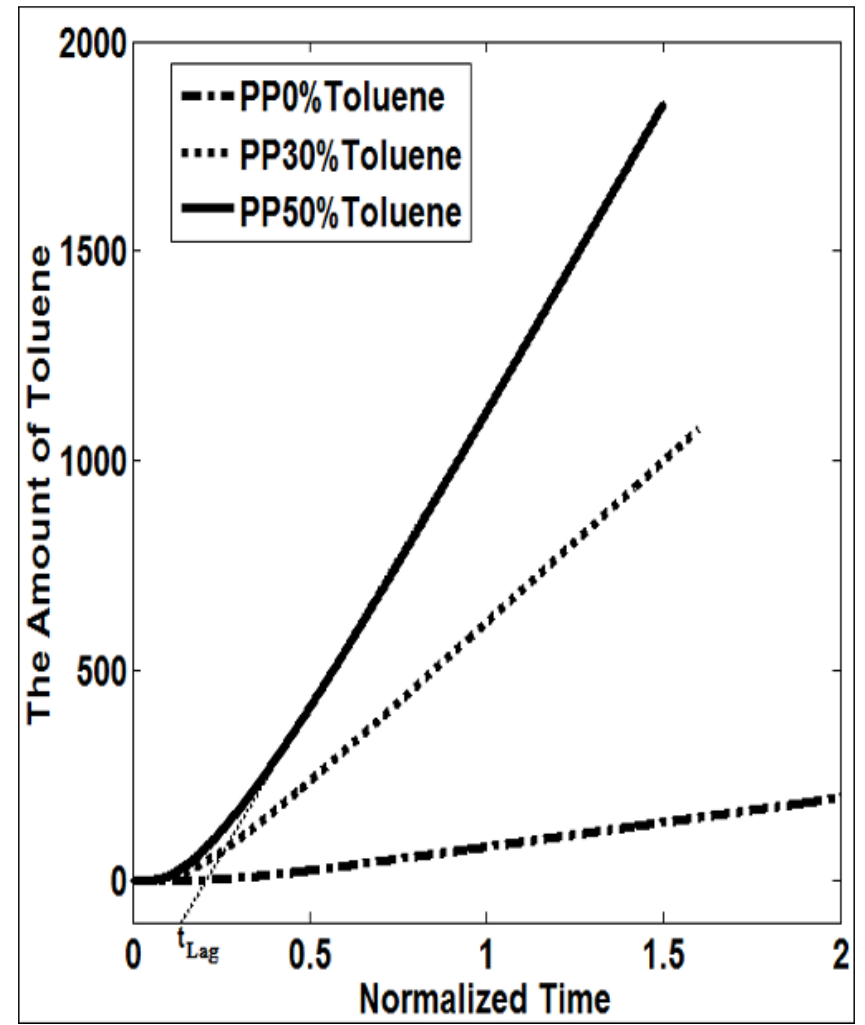

Figure 2. Global permeation mass flux vs normalized time for toluene in PP/NBR at different blend compositions.

In Figure 2, we show the integral form of the solvent mass flux at the exit of the polymeric blend $(X=1)$ against the normalized time. We determine the time lag, $t_{\text {Lag }}$ corresponding to the steady state of the diffusion mass flux. It is clearly observed that the time lag decreases with the volume fraction of PP within the immiscible blend. This is due among others to the decrease of the diffusivity coefficient of toluene in the blend as shown in Table 1.

\begin{tabular}{|l|l|l|l|}
\hline Blends & $\mathrm{PP}(0 \%)$ & $\mathrm{PP}(30 \%)$ & $\mathrm{PP}(50 \%)$ \\
\hline$D_{0}\left(\mathrm{~m}^{2} / \mathrm{s}\right)$ & $4,45.10^{-11}$ & $3,5.10^{-11}$ & $2,03.10^{-11}$ \\
\hline
\end{tabular}

Table 1. Diffusivity coefficient of toluene into PP/NBR immiscible blend at different compositions [3]

\section{Conclusion}

In this contribution, we investigate the implications of a non-Fickian model that takes into account the contribution of the microstructure on mass transport and reciprocally. The system, we have considered, is a mixture of two polymers that do not mix at the microscopic level and thus a macroscopic interface appears within the immiscible blend. The model governing equations incorporate the dynamic changes of the area (size and anisotropy) of the interface as well as of the conformation of macromolecular chains. We calculate the unsteady permeation mass flux of toluene into a PP/NBR immiscible blend at different compositions. Moreover, in this non-Fickian regime we were capable of predicting the time lag necessary for the solvent flux to reach its steady state. This is achieved by evaluating the global permeation mass flux at the exit of the immiscible blend.

\section{References}

[1] A. El Afif, R. Cortez, D. P. Gaver III and D. De Kee, Modeling of mass transport into immiscible polymeric blends, Macromolecules, Volume 36 (2003) 9216-9229.

[2] A. El Afif and M. El Omari, Flow and mass transport in blends of immiscible viscoelastic polymers, Rheol Acta, Volume 48 (2008) 285-299.

[3] S. George, K. T. Varughes, S. Thomas, Molecular transport of aromatic solvents in isotactic polypropylene/acrylonitrile-co-butadiene rubber blends, Polymer, Volume 41 (2000) 579-594. 